\title{
ON AN INEQUALITY OF DIANANDA
}

\section{PENG GAO}

\author{
Received 17 October 2002
}

We consider certain refinements of the arithmetic and geometric means. The results generalize an inequality of P. Diananda.

2000 Mathematics Subject Classification: 26D15.

1. Introduction. Let $P_{n, r}(\mathbf{x})$ be the generalized weighted means

$$
P_{n, r}(\mathbf{x})=\left(\sum_{i=1}^{n} q_{i} x_{i}^{r}\right)^{1 / r},
$$

where $P_{n, 0}(\mathbf{x})$ denotes the limit of $P_{n, r}(\mathbf{x})$ as $r \rightarrow 0^{+}$and where $q_{i}>0,1 \leq i \leq n$, are positive real numbers with $\sum_{i=1}^{n} q_{i}=1$ and $\mathbf{x}=\left(x_{1}, x_{2}, \ldots, x_{n}\right)$. In this note, we let $q=\min q_{i}$ and always assume $n \geq 2$ and $0 \leq x_{1}<x_{2}<\cdots<x_{n}$.

We define $A_{n}(\mathbf{x})=P_{n, 1}(\mathbf{x}), G_{n}(\mathbf{x})=P_{n, 0}(\mathbf{x})$, and $H_{n}(\mathbf{x})=P_{n,-1}(\mathbf{x})$ and we will write $P_{n, r}$ for $P_{n, r}(\mathbf{x}), A_{n}$ for $A_{n}(\mathbf{x})$, and similarly for other means when there is no risk of confusion.

For mutually distinct numbers $r, s$, and $t$ and any real number $\alpha$ and $\beta$, we define

$$
\Delta_{r, s, t, \alpha, \beta}=\left|\frac{P_{n, r}^{\alpha}-P_{n, t}^{\alpha}}{P_{n, r}^{\beta}-P_{n, s}^{\beta}}\right|,
$$

where we interpret $P_{n, r}^{0}-P_{n, s}^{0}$ as $\ln P_{n, r}-\ln P_{n, s}$. When $\alpha=\beta$, we define $\Delta_{r, s, t, \alpha}$ to be $\Delta_{r, s, t, \alpha, \alpha}$. As a limit case

$$
\Delta_{r, s, t, 0}=\lim _{\alpha \rightarrow 0} \Delta_{r, s, t, \alpha}=\left|\frac{\left(\ln \left(P_{n, r} / P_{n, t}\right)\right)}{\left(\ln \left(P_{n, r} / P_{n, s}\right)\right)}\right| .
$$

Bounds for $\Delta_{r, s, t, \alpha, \beta}$ have been studied by many mathematicians. For the case $\alpha \neq \beta$, we refer the reader to [2, 5, 7] for the detailed discussions. When $\alpha=\beta$, we can bound $\Delta_{r, s, t, \alpha}$ in terms of $r, s$, and $t$ only, due to the following result of Hsu [6] (see also [1]).

THEOREM 1.1. For $r>s>t>0$,

$$
1<\Delta_{r, s, t, 1}<\frac{s(r-t)}{t(r-s)} .
$$


It is also interesting to consider the following bounds:

$$
f_{r, s, t, \alpha}(q) \geq \Delta_{r, s, t, \alpha} \geq g_{r, s, t, \alpha}(q),
$$

where $f_{r, s, t, \alpha}(q)$ is a decreasing function of $q$ and $g_{r, s, t, \alpha}(q)$ is an increasing function of $q$.

The cases $r=1, s=0, t=-1, \alpha=0, q_{i}=1 / n, 1 \leq i \leq n$, in (1.5) with $f_{1,0,-1,0}(q)=n$ and $g_{1,0,-1,0}(q)=n /(n-1)$, are the famous Sierpiński's inequality [9].

Another cases, $r=1, s=1 / 2, t=0$, and $\alpha=1$ with $f_{1,1 / 2,0,1}(q)=1 / q$ and $g_{1,1 / 2,0,1}(q)=1 /(1-q)$ were proved by Diananda [3, 4] (see also [1, 8]), originally stated as

$$
\frac{1}{q} \Sigma_{n} \geq A_{n}-G_{n} \geq \frac{1}{1-q} \Sigma_{n}
$$

where $\Sigma_{n}=\sum_{1 \leq i<j \leq n} q_{i} q_{j}\left(x_{i}^{1 / 2}-x_{j}^{1 / 2}\right)^{2}$.

The main purpose of this note is to generalize Diananda's result, which is given by Theorem 3.1.

\section{Lemmas}

LEMMA 2.1. For $0 \leq q \leq 1 / 2$,

$$
\begin{gathered}
\frac{r-1}{r}-\left(1-q^{r-1}\right) \leq 0 \quad(r \geq 2), \\
\left|\frac{r-1}{r}\right| \geq\left|1-(1-q)^{r-1}\right| \quad(0<r \leq 2)
\end{gathered}
$$

with equality holding if and only if $r=2$ and $q=1 / 2$.

Proof. We prove (2.1) here and the proof for (2.2) is similar. It suffices to prove (2.1) for $q=1 / 2$, which is equivalent to $2^{r} \geq 2 r$. Notice that the two curves $y=2^{r}$ and $y=2 r$ only intersect at $r=1$ and $r=2$ in which cases they are equal and the conclusion then follows.

LEMMA 2.2. For $0<q \leq 1$, the function

$$
f(q)=\left|\frac{q}{1-(1-q)^{r-1}}\right|
$$

is decreasing for $0<r \neq 1<2$ and increasing for $r>2$.

Proof. We prove the case $1<r \neq 2$ here and the case $0<r<1$ is similar. We have

$$
f^{\prime}(q)=\frac{1-(1-q)^{r-1}-q(r-1)(1-q)^{r-2}}{\left(1-(1-q)^{r-1}\right)^{2}}
$$

and by the mean value theorem, $1-(1-q)^{r-1}=q(r-1) \eta^{r-2}$, where $1-q<$ $\eta<1$, which implies $f^{\prime}(q) \leq 0$ for $1<r<2$ and $f^{\prime}(q) \geq 0$ for $r>2$. 
LEMMA 2.3. For $0<r \neq 1<2$ and $0<q \leq 1 / 2$,

$$
\left|\frac{1 / 2}{1-1 / r}\right|<\left|\frac{q}{1-(1-q)^{r-1}}\right| \text {. }
$$

If $r>2,(2.5)$ is valid with "> " instead of " $<$ ".

Proof. We prove the case $1<r<2$ here and the other cases are similar. By Lemma 2.2, it suffices to show (2.5) for $q=1 / 2$. In this case, (2.5) is equivalent to (2.2).

\section{The main theorems}

THEOREM 3.1. For any $t \neq 0$,

$$
\begin{aligned}
& \Delta_{t, t / r, 0, t} \geq \frac{1}{1-q^{r-1}} \quad(r \geq 2), \\
& \Delta_{t, t / r, 0, t} \leq\left|\frac{1}{1-(1-q)^{r-1}}\right| \quad(0<r \neq 1 \leq 2)
\end{aligned}
$$

with equality holding if and only if $n=2, x_{1}=0$, and $q_{2}=q$ for (3.1) and $n=2, x_{1}=0$, and $q_{1}=q$ for (3.2), except in the trivial cases $r=n=2$ and $q_{1}=q_{2}=1 / 2$.

PROOF. Since the proofs of (3.1) and (3.2) are very similar, we only prove (3.1) here and we just point out that (2.2) is needed for the proof of (3.2). The case $r=2$ was treated in [3], so we will assume that $r>2$ from now on. First, consider the case $t=1$ and define

$$
D_{n}(\mathbf{x})=\left(1-q^{r-1}\right)\left(A_{n}-G_{n}\right)-\left(A_{n}-P_{n, 1 / r}\right)
$$

and then we have

$$
\frac{1}{q_{n}} \frac{\partial D_{n}}{\partial x_{n}}=\left(1-q^{r-1}\right)\left(1-\frac{G_{n}}{x_{n}}\right)-\left(1-\left(\frac{P_{n, 1 / r}}{x_{n}}\right)^{1-1 / r}\right) .
$$

By a change of variables: $x_{i} / x_{n} \rightarrow x_{i}, 1 \leq i \leq n$, we may assume $0 \leq x_{1}<$ $x_{2}<\cdots<x_{n}=1$ in (3.4) and rewrite it as

$$
g_{n}\left(x_{1}, \ldots, x_{n-1}\right):=\left(1-q^{r-1}\right)\left(1-G_{n}\right)-\left(1-\left(P_{n, 1 / r}\right)^{1-1 / r}\right) .
$$

We want to show that $g_{n} \geq 0$. Let $\mathbf{a}=\left(a_{1}, \ldots, a_{n-1}\right) \in[0,1]^{n-1}$ be the point in which the absolute minimum of $g_{n}$ is reached.

We may assume $a_{1} \leq a_{2} \leq \cdots \leq a_{n-1}$. If $a_{i}=a_{i+1}$ for some $1 \leq i \leq n-2$ or $a_{n-1}=1$, by combing $a_{i}$ with $a_{i+1}$ and $q_{i}$ with $q_{i+1}$ or $a_{n-1}$ with 1 and $q_{n-1}$ with $q_{n}$, we can reduce the determination of the absolute minimum of $g_{n}$ to 
that of $g_{n-1}$ with different weights. Thus, without loss of generality, we may assume $a_{1}<a_{2}<\cdots<a_{n-1}<1$. If $\mathbf{a}$ is a boundary point of $[0,1]^{n-1}$, then $a_{1}=0$ and (3.5) is reduced to

$$
g_{n}=1-q^{r-1}-\left(1-\left(P_{n, 1 / r}\right)^{1-1 / r}\right) .
$$

It follows that $g_{n} \geq 0$ is equivalent to $P_{n, 1 / r} \geq q^{r}$ while the last inequality is easily verified with equality holding if and only if $n=2, a_{1}=0$, and $q_{2}=q$. Thus $g_{n} \geq 0$ for this case.

Now we may assume $a_{1}>0$ and $\mathbf{a}$ is an interior point of $[0,1]^{n-1}$, then we obtain

$$
\nabla g_{n}\left(a_{1}, \ldots, a_{n-1}\right)=0
$$

such that $a_{1}, \ldots, a_{n-1}$ solve the equation

$$
-\left(1-q^{r-1}\right) \frac{G_{n}}{x}+\left(1-\frac{1}{r}\right)\left(P_{n, 1 / r}\right)^{-1 / r}\left(\frac{P_{n, 1 / r}}{x}\right)^{1-1 / r}=0 .
$$

The above equation has at most one root (regarding $G_{n}$ and $P_{n, 1 / r}$ as constants), so we only need to show that $g_{n} \geq 0$ for the case $n=2$. Now by letting $0<x_{1}=x<x_{2}=1$ in (3.5), we get

$$
\frac{1}{q_{1}} g_{2}^{\prime}(x)=h(x) x^{1 / r-1},
$$

where

$$
h(x)=\frac{r-1}{r}\left(q_{1} x^{1 / r}+q_{2}\right)^{r-2}-\left(1-q^{r-1}\right) x^{q_{1}-1 / r} .
$$

If $1 / r \geq q_{1}$, then

$$
\begin{aligned}
h^{\prime}(x)= & \frac{(r-1)(r-2)}{r^{2}} q_{1} x^{1 / r-1}\left(q_{1} x^{1 / r}+q_{2}\right)^{r-3} \\
& -\left(1-q^{r-1}\right)\left(q_{1}-\frac{1}{r}\right) x^{q_{1}-1 / r-1} \geq 0
\end{aligned}
$$

which implies

$$
h(x) \leq h(1)=\frac{r-1}{r}-\left(1-q^{r-1}\right)<0
$$

for $r>2$ and $q \leq 1 / 2$ by Lemma 2.1, and thus $g_{2}(x) \geq g_{2}(1)=0$.

If $q_{1}>1 / r$, we have

$$
\begin{aligned}
\lim _{x \rightarrow 0^{+}} h(x) & =\lim _{x \rightarrow 0^{+}}\left(\frac{r-1}{r}\left(q_{1} x^{1 / r}+q_{2}\right)^{r-2}-\left(1-q^{r-1}\right) x^{q_{1}-1 / r}\right)>0, \\
\lim _{x \rightarrow 1^{-}} h(x) & =\lim _{x \rightarrow 1^{-}}\left(\frac{r-1}{r}\left(q_{1} x^{1 / r}+q_{2}\right)^{r-2}-\left(1-q^{r-1}\right) x^{q_{1}-1 / r}\right) \\
& =\frac{r-1}{r}-\left(1-q^{r-1}\right)<0 .
\end{aligned}
$$


Notice here that any positive root of $h(x)$ also satisfies the equation

$$
P(x)=q_{1} x^{1 / r}+q_{2}-\left(C x^{q_{1}-1 / r}\right)^{1 /(r-2)}=0,
$$

where $C=r\left(1-q^{r-1}\right) /(r-1)$.

It is easy to see that $P^{\prime}(x)$ can have at most one positive root. Thus by Rolle's theorem, $P(x)$ hence $h(x)$ can have at most two roots in $(0,1)$. Equations (3.13) and (3.14) further imply $h(x)$, hence $g_{2}^{\prime}(x)$ has exactly one root $x_{0}$ in $(0,1)$. Since (3.14) shows that $g_{2}^{\prime}(1)<0, g_{2}(x)$ takes its maximum value at $x_{0}$. Thus $g_{2}(x) \geq \min \left\{g_{2}(0), g_{2}(1)\right\}=0$.

Thus we have shown $g_{n} \geq 0$, hence $\partial D_{n} / \partial x_{n} \geq 0$ with equality holding if and only if $n=1$ or $n=2, x_{1}=0$, and $q_{2}=q$. By letting $x_{n}$ tend to $x_{n-1}$, we have $D_{n} \geq D_{n-1}$ (with weights $q_{1}, \ldots, q_{n-2}, q_{n-1}+q_{n}$ ). Since $1-q^{r-1}$ is a decreasing function of $q$, it follows by induction that $D_{n}>D_{n-1}>\cdots>D_{2}=0$ when $x_{1}=0$ and $q_{2}=q$ in $D_{2}$ or else $D_{n}>D_{n-1}>\cdots>D_{1}=0$. Since we assume that $n \geq 2$ in this note, this completes the proof for $t=1$.

Now for an arbitrary $t$, a change of variables $x_{i} \rightarrow x_{i}^{t}$ in the above cases leads to the desired conclusion.

We remark here that the constants in (3.1) and (3.2) are best possible by considering the cases $n=2, x_{1}=0$, and $q_{2}=q$ or $q_{1}=q$. Also when $n=2$, we conclude from the proof of Lemma 2.1 and $\lim _{x_{1} \rightarrow x_{2}} \Delta_{t, t / r, 0, t}=r /(r-1)$ that an upper bound in the form of (3.2) does not hold for $\Delta_{1,1 / r, 0,1}$ when $r>2$. Similarly, a lower bound in the form of (3.1) does not hold for $1<r<2$.

For $t=1$, rewrite (3.1) as

$$
A_{n}-G_{n} \geq \frac{1}{1-q^{r-1}}\left(A_{n}-P_{n, 1 / r}\right)
$$

When $n=2$, we have

$$
\lim _{x_{1} \rightarrow x_{2}} \frac{\left(A_{2}-P_{2,1 / 2}\right) /(1-q)}{\left(A_{2}-P_{2,1 / r^{\prime}}\right) /\left(1-q^{r^{\prime}-1}\right)}=\frac{(1 / 2) /(1-q)}{\left(1-1 / r^{\prime}\right) /\left(1-q^{r^{\prime}-1}\right)} .
$$

By considering $q=0,1 / 2$, we find that the right-hand side of (3.16) is not comparable for $r=2$ and any $r^{\prime}>2$.

However, for the comparison of the left-hand side of (3.2), we have the following theorem.

THEOREM 3.2. For any $t \neq 0$ and $0<r \neq 1<2$,

$$
\left|\frac{q}{1-(1-q)^{r-1}}\right| \geq \Delta_{t, t / r, t / 2, t}
$$

If $r \geq 2$, (3.18) is valid with " $\leq$ " instead of " $\geq$ " with equality holding in all the cases if and only if $n=2, x_{1}=0$, and $q_{1}=q$. 
Proof. Since the proofs are similar, we only prove the case $1<r<2$ here. Notice by Lemma 2.2 that $q /\left(1-(1-q)^{r-1}\right)$ is decreasing with respect to $q$, so we can prove by induction as we did in the proof of Theorem 3.1. First consider the case $t=1$ and define

$$
E_{n}(\mathbf{x})=q\left(A_{n}-P_{n, 1 / r}\right)-\left(1-(1-q)^{r-1}\right)\left(A_{n}-P_{n, 1 / 2}\right),
$$

so

$$
\frac{1}{q_{n}} \frac{\partial E_{n}}{\partial x_{n}}=q\left(1-\left(\frac{P_{n, 1 / r}}{x_{n}}\right)^{1-1 / r}\right)-\left(1-(1-q)^{r-1}\right)\left(1-\left(\frac{P_{n, 1 / 2}}{x_{n}}\right)^{1 / 2}\right) .
$$

By a change of variables: $x_{i} / x_{n} \rightarrow x_{i}, 1 \leq i \leq n$, we may assume $0<x_{1}<$ $x_{2}<\cdots<x_{n}=1$ in (3.20) and rewrite it as

$$
h_{n}\left(x_{1}, \ldots, x_{n-1}\right):=q\left(1-\left(P_{n, 1 / r}\right)^{1-1 / r}\right)-\left(1-(1-q)^{r-1}\right)\left(1-P_{n, 1 / 2}^{1 / 2}\right) .
$$

We want to show that $h_{n} \geq 0$. Let $\mathbf{a}=\left(a_{1}, \ldots, a_{n-1}\right) \in[0,1]^{n-1}$ be the point in which the absolute minimum of $h_{n}$ is reached. Similar to the proof of Theorem 3.1, we may assume $a_{1}<a_{2}<\cdots<a_{n-1}<1$. If $\mathrm{a}$ is a boundary point of $[0,1]^{n-1}$, then $a_{1}=0$, and we can regard $h_{n}$ as a function of $a_{2}, \ldots, a_{n-1}$, then we obtain

$$
\nabla h_{n}\left(a_{2}, \ldots, a_{n-1}\right)=0
$$

Otherwise $a_{1}>0$, a is an interior point of $[0,1]^{n-1}$ and

$$
\nabla h_{n}\left(a_{1}, \ldots, a_{n-1}\right)=0 \text {. }
$$

In either cases, $a_{2}, \ldots, a_{n-1}$ solve the equation

$$
-q\left(1-\frac{1}{r}\right)\left(P_{n, 1 / r}\right)^{-1 / r}\left(\frac{P_{n, 1 / r}}{x}\right)^{1-1 / r}+\frac{1}{2}\left(1-(1-q)^{r-1}\right) x^{-1 / 2}=0 .
$$

The above equation has at most one root (regarding $P_{n, 1 / r}$ as a constant), so we only need to show $h_{n} \geq 0$ for the case $n=3$ with $0=x_{1}<x_{2}=x<x_{3}=1$ in (3.21). In this case we regard $h_{3}$ as a function of $x$ and we get

$$
\frac{1}{q_{2}} h_{3}^{\prime}(x)=-q \frac{r-1}{r}\left(q_{2} x^{1 / r}+q_{3}\right)^{r-2} x^{1 / r-1}+\frac{1}{2}\left(1-(1-q)^{r-1}\right) x^{-1 / 2} .
$$

Let $x$ be a critical point, then $h_{3}^{\prime}(x)=0$. Similar to the proof of Theorem 3.1, there can be at most two roots in $[0,1]$ for $h_{3}^{\prime}(x)=0$.

Further notice that

$$
\lim _{x \rightarrow 1^{-}} \frac{1}{q_{2}} h_{3}^{\prime}(x)=-q \frac{r-1}{r}\left(1-q_{1}\right)^{r-2}+\frac{1-(1-q)^{r-1}}{2}<0
$$


by Lemma 2.3 and

$$
\lim _{x \rightarrow 0^{+}} \frac{1}{q_{2}} h_{3}^{\prime}(x)=+\infty .
$$

It then follows that $h_{3}^{\prime}(x)$ has exactly one root $x_{0}$ in $(0,1)$ and $h_{3}^{\prime}(1)<0$ implies that $h_{3}(x)$ takes its maximum value at $x_{0}$. Thus

$$
h_{3}(x) \geq \min \left\{h_{3}(0), h_{3}(1)\right\} \geq 0,
$$

where the last inequality follows from Lemma 2.2. Thus $D_{n} \geq 0$ with equality holding if and only if $n=2, x_{1}=0$, and $q_{1}=q$, and a change of variables $x_{i} \rightarrow x_{i}^{t}$ completes the proof.

Notice here for $1<r<2$, by setting $t=1$ and letting $q \rightarrow 0$ in (3.18) while noticing that $q /\left(1-(1-q)^{r-1}\right)$ is a decreasing function of $q$, we get

$$
\Delta_{1,1 / r, 1 / 2,1} \leq \frac{1}{r-1},
$$

a special case of Theorem 1.1, which shows that, in this case, Theorem 3.2 refines Theorem 1.1.

We end the note by refining a result of the author [5].

THEOREM 3.3. If $x_{1} \neq x_{n}, n \geq 2$, then for $1>s \geq 0$ and $\sigma_{n, 1}=\sum_{i=1}^{n} q_{i}\left(x_{i}-\right.$ $\left.A_{n}\right)^{2}$,

$$
\begin{aligned}
& \frac{P_{n, s}^{1-s}-x_{1}^{1-s}}{2 x_{1}^{1-s}\left(A_{n}-x_{1}\right)} \sigma_{n, 1}-q \frac{\left(A_{n}-P_{n, s}\right)^{2}}{2\left(A_{n}-x_{1}\right)} \\
& \quad>A_{n}-P_{n, s}>\frac{x_{n}^{1-s}-P_{n, s}^{1-s}}{2 x_{n}^{1-s}\left(x_{n}-A_{n}\right)} \sigma_{n, 1}+q \frac{\left(A_{n}-P_{n, s}\right)^{2}}{2\left(x_{n}-A_{n}\right)} .
\end{aligned}
$$

Proof. We will prove the right-hand side inequality and the proofs for the left-hand side inequality are similar. Let

$$
F_{n}(\mathbf{x})=\left(x_{n}-A_{n}\right)\left(A_{n}-P_{n, s}\right)-\frac{x_{n}^{1-s}-P_{n, s}^{1-s}}{2 x_{n}^{1-s}} \sigma_{n, 1}-q \frac{\left(A_{n}-P_{n, s}\right)^{2}}{2} .
$$

We want to show by induction that $F_{n} \geq 0$. We have

$$
\begin{aligned}
\frac{\partial F_{n}}{\partial x_{n}}= & \left(1-q_{n}-q q_{n}\left(1-\left(\frac{P_{n, s}}{x_{n}}\right)^{1-s}\right)\right)\left(A_{n}-P_{n, s}\right) \\
& -\frac{1-s}{2 x_{n}}\left(\frac{P_{n, s}}{x_{n}}\right)^{1-s}\left(1-\left(\frac{x_{n}}{P_{n, s}}\right)^{s} q_{n}\right) \sigma_{n, 1} \\
\geq & \left(1-q_{n}\right)\left(\frac{P_{n, s}}{x_{n}}\right)^{1-s}\left(A_{n}-P_{n, s}-\frac{1-s}{2 x_{n}} \sigma_{n, 1}\right) \geq 0,
\end{aligned}
$$

where the last inequality holds by [5, Theorem 2.1]. Thus by a similar induction process as the one in the proof of Theorem 3.1, we have $F_{n} \geq 0$. Since not all the $x_{i}$ 's are equal, we get the desired result. 
Acknowledgment. The author is grateful to the referees for helpful comments and suggestions.

\section{REFERENCES}

[1] P. S. Bullen, D. S. Mitrinović, and P. M. Vasić, Means and Their Inequalities, Mathematics and Its Applications (East European Series), vol. 31, D. Reidel Publishing, Dordrecht, 1988.

[2] D. I. Cartwright and M. J. Field, A refinement of the arithmetic mean-geometric mean inequality, Proc. Amer. Math. Soc. 71 (1978), no. 1, 36-38.

[3] P. H. Diananda, On some inequalities of H. Kober, Proc. Cambridge Philos. Soc. 59 (1963), 341-346.

[4] _ _ "On some inequalities of H. Kober": Addendum, Proc. Cambridge Philos. Soc. 59 (1963), 837-839.

[5] P. Gao, Certain bounds for the differences of means, RGMIA Research Report Collection 5 (2002), no. 3, Article No. 7.

[6] L. C. Hsu, Questions 1843, 1844, Math. Student 23 (1955), 121.

[7] A. McD. Mercer, Some new inequalities involving elementary mean values, J. Math. Anal. Appl. 229 (1999), no. 2, 677-681.

[8] D. S. Mitrinović, J. E. Pečarić, and A. M. Fink, Classical and New Inequalities in Analysis, Mathematics and Its Applications (East European Series), vol. 61, Kluwer Academic Publishers Group, Dordrecht, 1993.

[9] W. Sierpiński, On an inequality for arithmetic, geometric and harmonic means, Warsch. Sitzungsber. 2 (1909), 354-358 (Polish).

Peng Gao: Department of Mathematics, University of Michigan, Ann Arbor, MI 48109, USA

E-mail address: penggao@umich.edu 


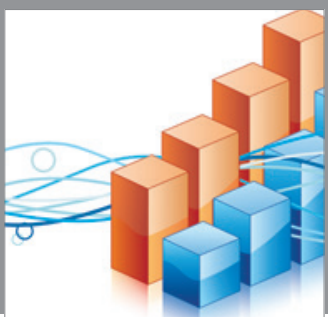

Advances in

Operations Research

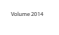

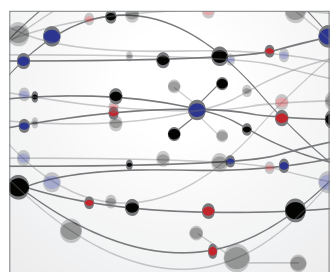

\section{The Scientific} World Journal
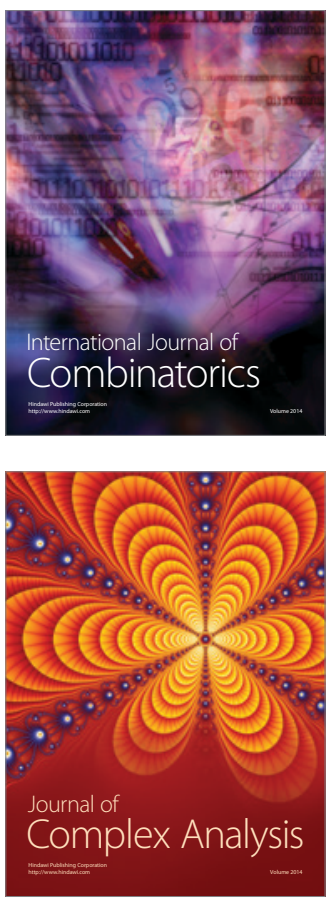

International Journal of

Mathematics and

Mathematical

Sciences
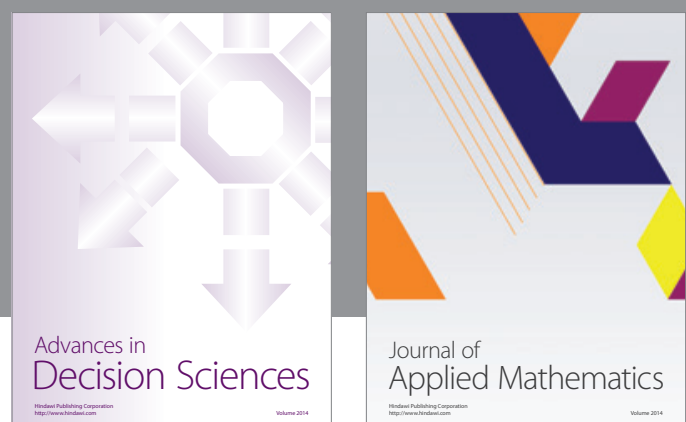

Journal of

Applied Mathematics
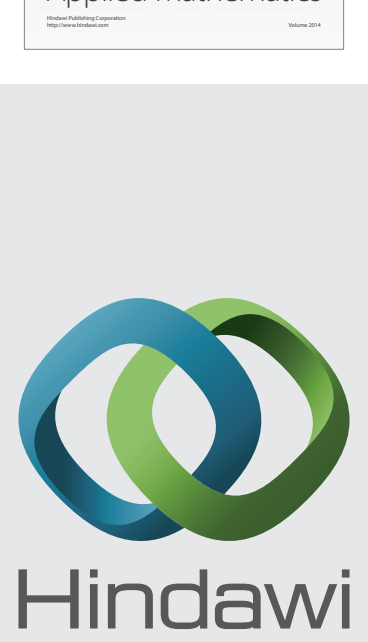

Submit your manuscripts at http://www.hindawi.com
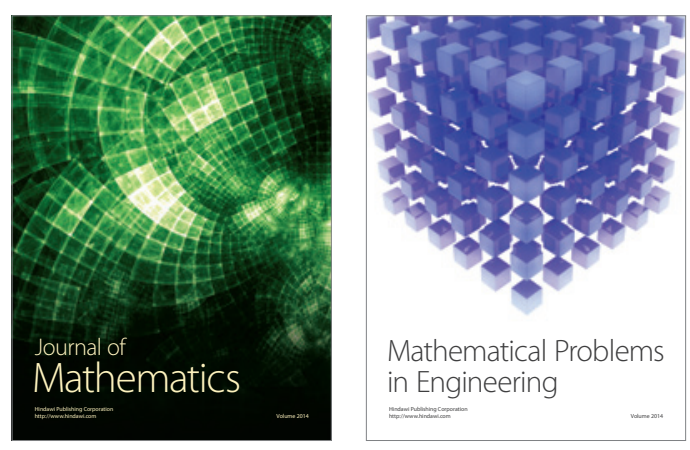

Mathematical Problems in Engineering
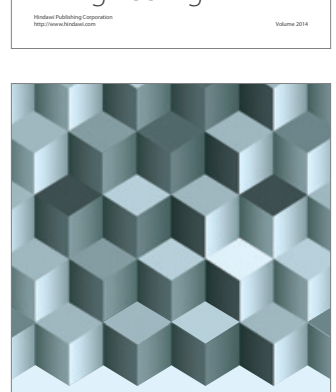

Journal of

Function Spaces
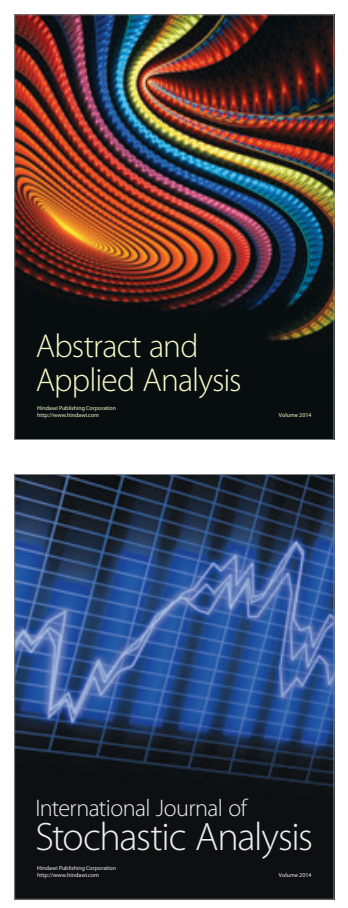

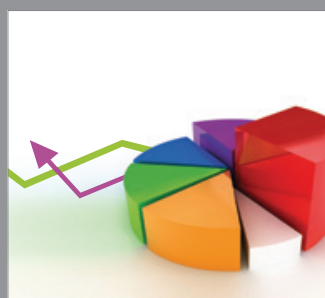

ournal of

Probability and Statistics

Promensencen
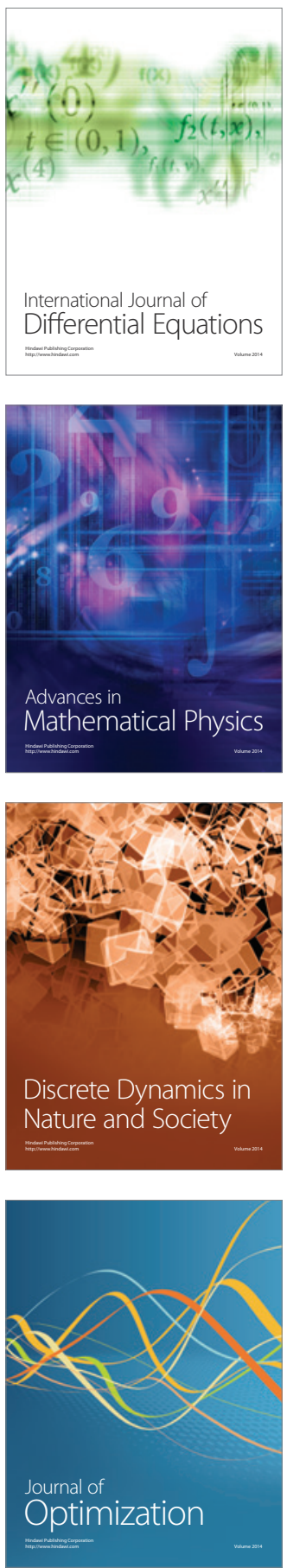